Decline' tool. Referrals were made by telephone to the Hospice Coordination Team. Band 3 carers were available to provide up to 24 hours care in patients' own homes dependant on need. There was ongoing review of care needs in liaison with the district nurse and hospice nurse specialists.

Results The service was effective and timely, supporting same or next day discharge. The service was responsive to patient and family need. The care provided was flexible, supporting patients in the last days of life alongside those awaiting a CHC Fast Track package of care. Good communication was fostered between the partner organisations. In one quarter 18 patients were discharged saving a total of 148 acute bed days. Conclusions The service has demonstrated a reduction in patient length of stay in the acute trust, improving patient flow in the wider health community. Funding has been secured for a further year and access will be extended to Discharge Liaison teams. Plans are now in place to address inequity across the patch and provide the service across other local authority areas.

\section{P-199 'EVERY DAY COUNTS' - PILOT OF A SERVICE FOR IMPROVING ACCESS AND CONSISTENCY IN PALLIATIVE CARE SEVEN DAYS A WEEK}

Christine Bloomer, Giovanna Cruz. Hospice Isle of Man, Douglas, Isle of Man, UK

10.1136/bmjspcare-2017-hospice.224

Background The National Institute for Health and Care Excellence (NICE) has highlighted the need for access to specialist palliative care seven days a week in order to improve the quality of care, experience and outcomes for patients and their families. (NICE, 2004; NICE, 2015) Little is known about the demand for a seven-day Palliative Care Clinical Nurse Specialist (PCCNS) service in a small island of $\sim 85000$ residents. Generalist services are available to patients on weekends and holidays.

Aim The aim of this pilot was to determine the need for, use of and the impact of a seven-day PCCNS service.

Method The pilot service was offered between 1 October 2016 and 31 March 2017 by the local hospice PCCNS team, the only specialist palliative care provider on the island. The service was established using the existing team of 5.2 full-time equivalent PCCNS, overseen by their manager. The PCCNS on duty for the weekend was available for urgent telephone calls and visits. For patients with anticipated need, contact was made as requested by PCCNS colleagues, and 'routine' work from the existing caseload was also undertaken. The service was publicised directly to existing patients, and health providers on the island. The number, source and reason for calls, visits and hospice or hospital admissions were recorded and analysed. Feedback was sought from patients, caregivers, the PCCNS team and health professionals.

Results The PCCNS team received 84 urgent calls, generating 59 visits and 113 additional calls. Main reasons for contact were for anxiety support (32\%), symptom management (28\%) and end of life care (16\%). Weekend service resulted in 12 in-patient admissions to hospice, $58 \%$ for end of life care.

Conclusion The number of contacts supports the case for weekend and bank holiday cover. A seven-day PCCNS service is feasible and beneficial to patients and their families.

\section{P-200 TRIAGE AND RAPID RESPONSE IN PALLIATIVE CARE: DOES IT MAKE A DIFFERENCE?}

Neil Grannon, Emer McKenna. Douglas Macmillan Hospice, Stoke-on-Trent, UK

\subsection{6/bmjspcare-2017-hospice.225}

Background There is national recognition that demand on palliative care services is undergoing rapid change. The shift in age demographics combined with increasing complexity requires a more adaptive approach to fulfilling the wishes of patients. NICE has previously suggested that as well as the traditional face to face visits during 9-5 that there should be telephone advice 24 hours a day. It goes on to say that face to face consultations outside of $9-5$, seven days a week is desirable. Consequently the community team has led an initiative to remodel the current service into one which 'Patients were seen at the right time, in the right place and by the right person.'

Aims

- Restructure the community team to enhance flexibility, deliver additional capacity, to be able to visit patients at any time of day or night

- The Community Team needs the flexibility to visit patients at any time of day or night

- Introduce triage for all new referrals to ascertain the actual patient need and the speed at which a response is needed

- Evaluate the change in outcomes.

Methods 1. Utilised change management and best practice tools to alter working patterns and responsiveness

2. A baseline review was conducted prior to implementation of triage. Post launch outcomes were reviewed results on a monthly basis to ensure relevant standards and targets had been adhered to.

Conclusion We have received positive feedback from staff and patients regarding the use and deployment of triage. Of the new referrals received in May 2017 we have identified 6\% of inappropriate referrals prior to first face to face meeting. In $3 \%$ of cases avoided inappropriate admissions to hospital and delivered an urgent response to $21 \%$ who previously may not have received a visit for 6.4 days

\section{P-201 THE HELPER SERVICE: VOLUNTEERS BRING COMPANIONSHIP AND A SENSE OF NORMALITY}

${ }^{1}$ Christine O'Sullivan, ${ }^{2}$ Debbie Hill. 'Marie Curie Hospice Liverpool, UK; ${ }^{2}$ Marie Curie, UK

10.1136/bmjspcare-2017-hospice.226

Background A scoping exercise undertaken by an end of life care charity revealed that people living with a terminal illness can be socially isolated, navigating the end of life journey can be complex and bewildering, carers can find the situation overwhelming and may long for respite. Many carers also feel isolated in bereavement.

Aim The Helper volunteer role was created in response to this feedback, with pilot services set up in 2009. Volunteers were trained to offer companionship and emotional support to people living with any terminal illness and their families, and to combat the issues listed above. 